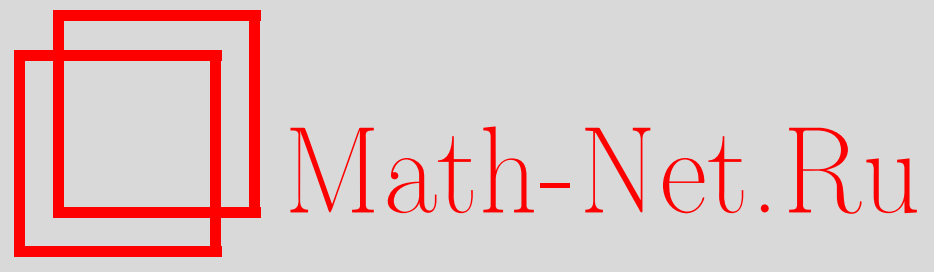

В. С. Романюк, Нелинейные поперечники классов гладких функций, определенных на единичной сфере в $\mathbb{R}^{d}$, Матем. заметки, 2009, том 85, выпуск 1, 147-152

DOI: https://doi.org/10.4213/mzm4152

Использование Общероссийского математического портала Math-Net.Ru подразумевает, что вы прочитали и согласны с пользовательским соглашением http://www . mathnet.ru/rus/agreement

Параметры загрузки:

IP : 3.91 .87 .62

26 апреля 2023 г., 13:13:06

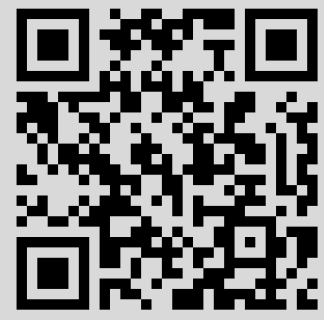




\section{Нелинейные поперечники классов гладких функций, определенных на единичной сфере в $\mathbb{R}^{d}$}

\section{В. С. Романюк}

В работе решается задача о нахождении слабой асимптотики одного типа нелинейных поперечников классов функций $W_{p}^{r}\left(\mathbb{S}^{d-1}\right)$, определенных на единичной сфере $\mathbb{S}^{d-1}$ пространства $\mathbb{R}^{d}$.

Пусть $\mathbb{R}^{d}, d \geqslant 3,-$ эвклидово пространство точек $\mathbf{x}=\left(x_{1}, \ldots, x_{d}\right)$,

$$
\mathbb{S}^{d-1}=\left\{\mathbf{x} \in \mathbb{R}^{d}:\|\mathbf{x}\|:=\left(x_{1}^{2}+\cdots+x_{d}^{2}\right)^{1 / 2}=1\right\},
$$

$L_{p}=L_{p}\left(\mathbb{S}^{d-1}\right), 1 \leqslant p \leqslant \infty,-$ пространство функций, определенных на $\mathbb{S}^{d-1}$ с конечной нормой

$$
\|f\|_{p}=\left\{\frac{1}{\Omega} \int_{\mathbb{S}^{d-1}}|f(\mathbf{x})|^{p} d \sigma(\mathbf{x})\right\}^{1 / p}, \quad 1 \leqslant p<\infty,
$$

где $d \sigma$ - поверхностная мера Лебега, $\Omega$ - площадь поверхности сферы $\mathbb{S}^{d-1} ; L_{\infty}$ отождествляется с пространством непрерывных на $\mathbb{S}^{d-1}$ функций с нормой

$$
\|f\|_{\infty}=\max _{\mathbf{x} \in \mathbb{S}^{d-1}}|f(\mathbf{x})| .
$$

Пусть $\mathscr{P}_{k}$ обозначает пространство алгебраических полиномов степени, не превышающей $k$, от $d$ переменных; $H_{k}^{d}$ - пространство однородных гармонических полиномов степени $k$ от $d$ переменных. Сужение элементов пространства $H_{k}^{d}$ на сферу $\mathbb{S}^{d-1}$ (сферические гармоники) образуют пространство, которое обозначается через $\mathscr{H}_{k}^{d}[1]$. Каждое подпространство $\mathscr{H}_{k}^{d}, k=0,1, \ldots$, является подпространством собственных функций оператора $\Delta_{0}$ Лапласа-Бельтрами на сфере (см., например, [2; с. 240]), т.е. для $Y \in \mathscr{H}_{k}^{d}$ $\Delta_{0} Y(\mathbf{x})=-k(k+d-2) Y(\mathbf{x}), \mathbf{x} \in \mathbb{S}^{d-1}$, и

$$
n_{k}:=\operatorname{dim} \mathscr{H}_{k}^{d}=(2 k+d-2) \frac{(k+d-3) !}{k !(d-2) !}, \quad k \geqslant 1, \quad n_{0}:=\operatorname{dim} \mathscr{H}_{0}^{d}=1 .
$$

Далее $L_{2}\left(\mathbb{S}^{d-1}\right)=\oplus \sum_{k=0}^{\infty} \mathscr{H}_{k}^{d}$, т.е. произвольная функция $f \in L_{2}\left(\mathbb{S}^{d-1}\right)$ однозначно представляется в $L_{2}\left(\mathbb{S}^{d-1}\right)$ в виде ряда по сферическим гармоникам

$$
f(\mathbf{x})=\sum_{k=0}^{\infty} Y_{k}(f ; \mathbf{x})=\sum_{k=0}^{\infty} \sum_{l=1}^{n_{k}} a_{k, l} Y_{k}^{l}(\mathbf{x}),
$$

где $\left\{Y_{k}^{l}\right\}_{l=1}^{n_{k}}$ - ортонормированный базис в $\mathscr{H}_{k}^{d}, a_{k, l}$ - коэффициенты Фурье-Лапласа функции $f$. Полагаем далее $\mathscr{H}(N)=\oplus \sum_{k=0}^{N} \mathscr{H}_{k}^{d}$.

На $\mathbb{S}^{d-1}$ можно определить операцию свертки [3]

$$
[f * g](\mathbf{x}):=\frac{1}{\Omega} \int_{\mathbb{S}^{d-1}} f(\mathbf{y}) g(\langle\mathbf{x}, \mathbf{y}\rangle) d \sigma(\mathbf{y}), \quad \mathbf{x} \in \mathbb{S}^{d-1},
$$

где $\langle\mathbf{x}, \mathbf{y}\rangle:=x_{1} y_{1}+\cdots+x_{d} y_{d}-$ скалярное произведение $\mathbf{x}, \mathbf{y} \in \mathbb{R}^{d}$, посредством которого представляется результат $Y_{k}(f ; \mathbf{x})$ действия оператора ортогонального проектирования $Y_{k}: L_{2}\left(\mathbb{S}^{d-1}\right) \rightarrow \mathscr{H}_{k}^{d}:$

$$
Y_{k}(f ; \mathbf{x})=\frac{1}{\Omega} \cdot \frac{k+\lambda}{\lambda} \int_{\mathbb{S}^{d-1}} f(\mathbf{y}) P_{k}^{\lambda}(\langle\mathbf{x}, \mathbf{y}\rangle) d \sigma(\mathbf{y}) .
$$

(C) B. С. Романюк, 2009 
Исходя из этого, определяются основные объекты, изучаемые в настоящей заметке: для $1 \leqslant p \leqslant \infty$ и $r>0$ положим

$$
W_{p}^{r}\left(\mathbb{S}^{d-1}\right):=\left\{f: f(\mathbf{x})=\left[\varphi * g_{r}\right](\mathbf{x}),\|\varphi\|_{p} \leqslant 1, \mathbf{x} \in \mathbb{S}^{d-1}\right\},
$$

где для $r>0$

$$
g_{r}(t)=\sum_{k=1}^{\infty}[k(k+d-2)]^{-r / 2} \frac{k+\lambda}{\lambda} P_{k}^{\lambda}(t), \quad \lambda=\frac{d-2}{2},
$$

а $P_{k}^{\lambda}(t),-1 \leqslant t \leqslant 1,-$ многочлены Гегенбауэра степени $k$ и индекса $\lambda$ (см., например, [4]) или ультрасферические многочлены, образующие, как известно, полную ортогональную систему в пространстве $L_{2}\left([-1 ; 1], w_{\lambda}\right)$ - квадратично-суммируемых с весом $w_{\lambda}(t)=$ $\left(1-t^{2}\right)^{\lambda-1 / 2}$ на $[-1,1]$ функций. В дальнейшем вместо $W_{p}^{r}\left(\mathbb{S}^{d-1}\right)$ будем писать $W_{p}^{r}$.

Нелинейным $n$-поперечником множества $F$ в банаховом пространстве $\mathscr{X}$ с нормой $\|\cdot\| \mathscr{X}$, определенным в [5], называется величина

$$
\bar{d}_{n}(F ; \mathscr{X})=\inf _{a, \mathscr{M}_{n}} \sup _{f \in F}\left\|f-M_{n}(a(f))\right\|_{\mathscr{X}},
$$

где $a: F \rightarrow \mathbb{R}^{n}$ - произвольное непрерывное отображение из $F$ в $\mathbb{R}^{n} ; \mathscr{M}_{n}$ - совокупность всех непрерывных отображений $M_{n}: \mathbb{R}^{n} \rightarrow \mathscr{X}$.

Для поперечника $\bar{d}_{n}(F ; \mathscr{X})$ известны следующие базовые соотношения с другими поперечниками:

$$
\begin{gathered}
\bar{d}_{2 n+1}(F ; \mathscr{X}) \leqslant a_{n}(F ; \mathscr{X}) \leqslant \bar{d}_{n}(F ; \mathscr{X}), \\
\bar{d}_{n}(F ; \mathscr{X}) \geqslant b_{n}(F ; \mathscr{X}) .
\end{gathered}
$$

Здесь $a_{n}(F ; \mathscr{X})$ и $b_{n}(F ; \mathscr{X})$ соответственно $n$-поперечники по Александрову и по Берштейну. Их определения и свойства можно найти, например, в книге [6]. Отметим, что неравенства (1) доказаны в [7], а неравенство (2) в [5].

Доказано следующее утверждение.

Tеорема. Пусть $r>0,1 \leqslant q \leqslant p \leqslant \infty$ uли $r>(d-1)^{2}+(d-1)(1 / p-1 / q), 1 \leqslant$ $p<q \leqslant \infty$. Тогда

$$
\bar{d}_{n}\left(W_{p}^{r} ; L_{q}\right) \asymp n^{-r /(d-1)} .^{1}
$$

Доказательство. Оценка сверху. Рассмотрим случай $1 \leqslant p<q \leqslant \infty$. Пусть $\eta-$ функция бесконечно-дифференцируемая на $(0, \infty)$ и такая, что $\operatorname{supp} \eta \subset[0 ; 2], \eta(x)=1$ при $0 \leqslant x \leqslant 1$, и $\eta(x)=0$ при $x \geqslant 2$. Зададим последовательность операторов $\left\{V_{n}\right\}_{n=1}^{\infty}$, определенных на множестве $L_{1}\left(\mathbb{S}^{d-1}\right)$ и действующих согласно формуле

$$
\left[V_{n}(f)\right](\mathbf{x})=\sum_{k=0}^{\infty} \eta\left(\frac{k}{n}\right) Y_{k}(f ; \mathbf{x}) .
$$

В качестве операторов $V_{n}$ можно рассматривать конкретные линейные средние рядов Фурье-Лапласа функции $f$ (см. [8]).

Положим

$$
\Phi_{0}(f)=V_{1}(f), \quad \Phi_{k}(f)=V_{2^{k}}(f)-V_{2^{k-1}}(f), \quad k=1,2, \ldots
$$

Тогда в силу свойств операторов $V_{n}$ любая функция $f \in L_{q}\left(\mathbb{S}^{d-1}\right), 1 \leqslant q \leqslant \infty$, представима в виде $f=\sum_{k=0}^{\infty} \Phi_{k}(f)$ (ряд сходится по норме $\|\cdot\|_{q}$ ), причем для $f \in L_{q}\left(\mathbb{S}^{d-1}\right.$ )

$$
\Phi_{k}(f) \in \mathscr{P}_{2^{k+1}-1}^{q}:=\mathscr{P}_{2^{k+1}-1} \cap L_{q}\left(\mathbb{S}^{d-1}\right), \quad k=0,1,2, \ldots
$$

\footnotetext{
${ }^{1}$ Для положительных последовательностей $\left(a_{n}\right)_{n=1}^{\infty}$ и $\left(b_{n}\right)_{n=1}^{\infty}$ запись $a_{n} \ll b_{n}$ означает, что существует постоянная $c>0$ такая, что $a_{n} \leqslant c b_{n}$ для любого $n \in \mathbb{N}$. Запись $a_{n} \asymp b_{n}$ равносильна тому, что $a_{n} \ll b_{n}$ и $b_{n} \ll a_{n}$.
} 
K тому же, если $f \in W_{p}^{r}, r>(d-1)(1 / p-1 / q)$, то согласно оценке величины наилучшего приближения класса $W_{p}^{r}$ в пространстве $L_{q}\left(\mathbb{S}^{d-1}\right)$, установленной в [9], в сочетании со свойствами операторов $V_{n}$ [10] (эти операторы реализуют такую оценку)

$$
\left\|\Phi_{k}(f)\right\|_{q} \ll\left(2^{k}\right)^{-r+(d-1)(1 / p-1 / q)_{+}}, \quad k=1,2, \ldots .
$$

Отправным при установлении оценки сверху величин $\bar{d}_{n}\left(W_{p}^{r} ; L_{q}\right)$ с учетом (1) является соотношение

$$
a_{n}\left(W_{p}^{r} ; L_{q}\right) \ll \sum_{j=0}^{j_{0}} a_{n_{j}}\left(\Phi_{j}\left(W_{p}^{r}\right) ; \mathscr{P}_{2^{j+1}-1}^{q}\right)+\sum_{j=j_{0}+1}^{\infty} d^{0}\left(\Phi_{j}\left(W_{p}^{r}\right) ; \mathscr{P}_{2^{j+1}-1}^{q}\right),
$$

справедливое для любых $n, j_{0}=0,1, \ldots$ и $\left\{n_{j}\right\}_{j=0}^{j_{0}}$ таких, что $\sum_{j=0}^{j_{0}} n_{j} \leqslant n$. Здесь $d^{n}(F ; \mathscr{X})$ (при $n=0$ ) обозначает поперечник по Гельфанду множества $F$ в пространстве $\mathscr{X}[6]$. Неравенство (4) следует из утверждения (доказанного в [11]) о промежуточной оценке копоперечников по Александрову $a^{n}(K ; \mathscr{X})$ компактного множества $K$ в банаховом пространстве $\mathscr{X}$ (определение см. в [6]) и из неравенства

$$
\frac{1}{4} a_{n}(K ; \mathscr{X}) \leqslant a^{n}(K ; \mathscr{X}) \leqslant 2 a_{n}(K ; \mathscr{X})
$$

(см. [6]).

Для продолжения оценки правой части (4) далее будем использовать весовой аналог теоремы Марцинкевича-Зигмунда (в частичной формулировке).

Tеорема A [12]. Существуют $m_{N}=(4 N+1)(2 N)^{d-2}$ точек $\xi_{N, 1}, \xi_{N, 2}, \ldots, \xi_{N, m_{N}}$ на сфере $\mathbb{S}^{d-1}$ таких, что для любого $f \in \mathscr{P}_{N} u 1 \leqslant p \leqslant \infty$

$$
\left(\int_{\mathbb{S}^{d-1}}|f(\mathbf{x})|^{p} d \sigma(\mathbf{x})\right)^{1 / p} \asymp\left(\frac{1}{m_{N}} \sum_{k=1}^{m_{N}} w_{N, k}\left|f\left(\xi_{N, k}\right)\right|^{p}\right)^{1 / p},
$$

где веса $w(N)=\left\{w_{N, k}\right\}_{k=1}^{m_{N}}$ удовлетворяют условиям $0<w_{N, k} \leqslant 1, w_{N, k}^{-1} \leqslant C(d) N^{(d-2)^{2}}$.

Исходя непосредственно из определения набора $\left\{w_{N, k}\right\}_{k=1}^{m_{N}}$ несложно показать (см. доказательство теоремы А в [12], а также замечания 5 и 6 в этой работе), что набор весов $w(N)$ обладает следующими свойствами, помимо приведенных в теореме А.

ПредлоЖение 1. Справедливъ утверждения:

(1) если $L \in \mathbb{N}$ - произвольное фиксированное, $M_{L}^{(j)}:=m_{L \cdot 2^{j}}, j=1,2, \ldots, \widetilde{M}_{L}^{(j)} \asymp$ $M_{L}^{(j)} \cdot 2^{-j(d-1)}$, то для любого набора $\widetilde{\Lambda}=\left\{k_{1}, \ldots, k_{\widetilde{M}_{L}^{(j)}}\right\}$ существует постоянная $C(d)>0$ такая, что

$$
\sum_{k \in \widetilde{\Lambda}} w_{L \cdot 2^{j}, k} \leqslant C(d) L^{d-1} \cdot 2^{-j(d-2)^{2}}
$$

(2) если $\Lambda=\left\{k_{1}, \ldots, k_{\widetilde{M}_{N}}\right\}$ u $\widetilde{M}_{N} \asymp m_{N}, m o$

$$
\sum_{k \in \Lambda} w_{N, k} \asymp N^{d-1}
$$

Продолжим преобразование правой части соотношения (4). Для $j=1,2, \ldots$ определим оператор $A_{j}: \mathscr{P}_{N_{j}} \rightarrow \mathbb{R}^{m_{N_{j}}}$ по формуле

$$
A_{j} f=\left(f\left(\xi_{N_{j}, 1}\right), \ldots, f\left(\xi_{N_{j}, m_{N_{j}}}\right)\right), \quad N_{j}=2^{j+1}-1, \quad j=0,1, \ldots,
$$


a $\left\{\xi_{N_{j}, k}\right\}_{k=1}^{m_{N_{j}}}-$ последовательность точек из теоремы А. Из теоремы А следует, что при $1 \leqslant q \leqslant \infty$ и $f \in \mathscr{P}_{\mathscr{N}_{j}}$

$$
\|f\|_{q} \asymp m_{N_{j}}^{-1 / q}\left\|A_{j} f\right\|_{l_{q, w\left(N_{j}\right)}^{m_{N_{j}}}},
$$

что вместе с неравенством (3) позволяет записать

$$
a_{n_{j}}\left(\Phi_{j}\left(W_{p}^{r}\right) ; \mathscr{P}_{2^{j+1}-1}^{q}\right) \ll N_{j}^{-r} m_{N_{j}}^{1 / p-1 / q} a_{n_{j}}\left(b_{p, w\left(N_{j}\right)}^{m_{N_{j}}} ; l_{q, w\left(N_{j}\right)}^{m_{N_{j}}}\right), \quad j=0,1, \ldots, j_{0} .
$$

Здесь для системы весов $w=\left\{w_{i}\right\}_{i=1}^{m}$ через $l_{p, w}^{m}$ обозначено пространство точек $\mathbf{x}=$ $\left(x_{1}, \ldots, x_{m}\right) \in \mathbb{R}^{m}$ с нормой

$$
\begin{array}{rlrl}
\|\mathbf{x}\|_{p, w} & =\left(\sum_{i=1}^{m}\left|x_{i}\right|^{p} w_{i}\right)^{1 / p}, & 1 \leqslant p<\infty, \\
\|\mathbf{x}\|_{\infty, w}=\max _{1 \leqslant i \leqslant m}\left|x_{i}\right| w_{i}, & p=\infty,
\end{array}
$$

$b_{p, w}^{m}:=\left\{\mathbf{x} \in l_{p, w}^{m}:\|\mathbf{x}\|_{p, w} \leqslant 1\right\}$. В случае $w_{i}=1, i=1, \ldots, m$, обозначение $l_{p, w}^{m}$ заменяем на $l_{p}^{m}$.

Далее, исходя из неравенства (4), на основании общих свойств поперечников $a_{n}$ и $d^{n}$ (см. например, [6; с. 217-218]) и соотношения (5), приходим к неравенству

$$
a_{n}\left(W_{p}^{r} ; L_{q}\right) \ll \sum_{j=0}^{j_{0}} N_{j}^{-r} m_{N_{j}}^{1 / p-1 / q} a_{n_{j}}\left(b_{p, w\left(N_{j}\right)}^{m_{N_{j}}} ; l_{q, w\left(N_{j}\right)}^{m_{N_{j}}}\right)+\sum_{j=j_{0}+1}^{\infty} N_{j}^{-r} m_{N_{j}}^{1 / p-1 / q}
$$

(при переходе от неравенства (4) к (6) мы заранее полагаем $n_{0}$ равным $m_{N_{0}}=5 \cdot 2^{d-2}$, а в этом случае при $j=0$ имеем $\left.a_{n_{j}}\left(\Phi_{j}\left(W_{p}^{r}\right) ; \mathscr{P}_{2^{j+1}-1}^{q}\right)=0\right)$.

Но непосредственно из определения поперечников $a_{k}$ можно записать

$$
a_{n_{j}}\left(b_{p, w\left(N_{j}\right)}^{m_{N_{j}}} ; l_{q, w\left(N_{j}\right)}^{m_{N_{j}}}\right)=a_{n_{j}}\left(B_{p}^{m_{N_{j}}}\left(\alpha\left(N_{j}\right)\right) ; l_{q}^{m_{N_{j}}}\right),
$$

где для заданного $\alpha=\left(\alpha_{1}, \ldots, \alpha_{m}\right), \alpha_{i}>0, i=1,2, \ldots, m$,

$$
B_{p}^{m}(\alpha):=\left\{\mathrm{x} \in \mathbb{R}^{m}: \sum_{i=1}^{m}\left|\frac{x_{i}}{\alpha_{i}}\right|^{p} \leqslant 1\right\}
$$

- $p$-эллипсоид в $l_{p}^{m}$, и при каждом $j=0,1, \ldots$ наборы $w\left(N_{j}\right)=\left\{w_{N_{j}, k}\right\}_{k=1}^{m_{N_{j}}}$ и $\alpha\left(N_{j}\right):=$ $\left\{\alpha_{N_{j}, k}\right\}_{k=1}^{m_{N_{j}}}$ связаны соотношением

$$
w_{N_{j}, k}=\alpha_{N_{j}, k}^{p q /(p-q)} \quad \text { или } \quad \alpha_{N_{j}, k}=w_{N_{j}, k}^{1 / q-1 / p}, \quad k=1,2, \ldots, m_{N_{j}} .
$$

Таким образом, следствием соотношения (6) с учетом (7) является неравенство

$$
a_{n}\left(W_{p}^{r} ; L_{q}\right) \ll \sum_{j=0}^{j_{0}} N_{j}^{-r} m_{N_{j}}^{1 / p-1 / q} a_{n_{j}}\left(B_{p}^{m_{N_{j}}}\left(\alpha\left(N_{j}\right)\right) ; l_{q}^{m_{N_{j}}}\right)+\sum_{j=j_{0}+1}^{\infty} N_{j}^{-r} m_{N_{j}}^{1 / p-1 / q} .
$$

Теперь по заданному (достаточно большому) $n \in \mathbb{N}$ найдем $s \in \mathbb{N}$ такое, что

$$
\sum_{j=0}^{s} m_{N_{j}}<n \leqslant \sum_{j=0}^{s+1} m_{N_{j}}
$$


(напомним, что $N_{j}=2^{j+1}-1$ и $\left.m_{N_{j}}=\left(4 N_{j}+1\right)\left(2 N_{j}\right)^{d-2} \asymp 2^{j(d-1)}\right)$ и положим в соотношении (8) $j_{0}=2 s$, а также $n_{j}=m_{N_{j}}$ при $0 \leqslant j \leqslant s$, и $n_{j}=N_{s}^{d-1} \cdot 2^{(s-j)(d-1)}$ при $s<j \leqslant 2 s$. Тогда

$$
\sum_{j=0}^{j_{0}} n_{j} \ll \sum_{j=0}^{s} 2^{j(d-1)}+\sum_{j=s+1}^{2 s} 2^{(2 s-j)(d-1)} \asymp 2^{s(d-1)} \asymp n .
$$

Далее понятно, что при $0 \leqslant j \leqslant s$ выполнено $a_{n_{j}}\left(B_{p}^{m_{N_{j}}}\left(\alpha\left(N_{j}\right)\right) ; l_{q}^{m_{N_{j}}}\right)=0$, а при $s<j \leqslant 2 s$ следствием точных значений поперечников $a_{k}\left(B_{p}^{m}(\alpha) ; l_{q}^{m}\right), 1 \leqslant p \leqslant q \leqslant \infty[6$; c. 232], с учетом предложения 1, является оценка

$$
a_{n_{j}}\left(B_{p}^{m_{N_{j}}}\left(\alpha\left(N_{j}\right)\right) ; l_{q}^{m_{N_{j}}}\right) \ll\left[N_{s}^{d-1} \cdot 2^{(s-j)(d-1)^{2}}\right]^{1 / q-1 / p} .
$$

Заметив, также, что

$$
\sum_{j=j_{0}+1}^{\infty} N_{j}^{-r} m_{N_{j}}^{1 / p-1 / q} \ll 2^{-s r} \asymp n^{-r /(d-1)}
$$

при $r>(d-1)(1 / p-1 / q)$, из (8) после несложных вычислений окончательно получаем, что если $1 \leqslant p<q \leqslant \infty$ и $r>(d-1)^{2}+(d-1)(1 / p-1 / q)$, то $a_{n}\left(W_{p}^{r} ; L_{q}\right) \ll n^{-r /(d-1)}$.

В случае $1 \leqslant p=q \leqslant \infty, r>0$ последняя оценка является следствием соотношения [9] $E_{n}\left(W_{p}^{r}\right)_{q} \asymp n^{-r+(d-1)(1 / p-1 / q)_{+}}$и цепочки неравенств

$$
a_{n}\left(W_{p}^{r} ; L_{q}\right) \leqslant d_{n}\left(W_{p}^{r} ; L_{q}\right) \ll E_{k}\left(W_{p}^{r}\right)_{q},
$$

в которой $n=\operatorname{dim} \mathscr{H}(k) \asymp k^{d-1}$, т.е. $k \asymp n^{1 /(d-1)}$.

Неравенство $a_{n}\left(W_{p}^{r} ; L_{q}\right) \ll n^{-r /(d-1)}$ сохраняется и в случае $1 \leqslant q<p \leqslant \infty$ вследствие вложения $L_{p} \subset L_{q}$, поскольку тогда $a_{n}\left(W_{p}^{r} ; L_{q}\right) \leqslant a_{n}\left(W_{p}^{r} ; L_{p}\right)$.

Наконец, приведенные выше оценки сверху для поперечника $a_{n}\left(W_{p}^{r} ; L_{q}\right)$ остаются справедливыми в силу соотношения (1) и для поперечника $\bar{d}_{n}$.

Оценка снизу. Пусть вначале $1 \leqslant p \leqslant q \leqslant \infty$. Рассмотрим оператор $\mathscr{D}^{r}, r>0$, "обобщенного" дифференцирования на сфере $\mathbb{S}^{d-1}$, определив его как мультипликаторный оператор, действующий по формуле

$$
Y_{k}\left(\mathscr{D}^{r} f ; \mathbf{x}\right)=[k(k+d-2)]^{r / 2} Y_{k}(f ; \mathbf{x}), \quad f \in L_{1}\left(\mathbb{S}^{d-1}\right), \quad k=0,1, \ldots
$$

Тогда для полинома $F(\mathbf{x})=\sum_{k=0}^{N} Y_{k}(\mathbf{x}), Y_{k} \in \mathscr{H}_{k}^{d}$, справедливо равенство

$$
\mathscr{D}^{r} F(\mathbf{x})=\sum_{k=1}^{N}[k(k+d-2)]^{r / 2} Y_{k}(\mathbf{x}), \quad \mathbf{x} \in \mathbb{S}^{d-1} .
$$

В [13] доказано, что для производных $\mathscr{D}^{r} F$ полиномов по сферическим гармоникам степени, не превышающей $N$, имеет место неравенство Бернштейна $\left\|\mathscr{D}^{r} F\right\|_{p} \ll N^{r}\|F\|_{q}$, $1 \leqslant p \leqslant q \leqslant \infty$. Согласно этому неравенству $b_{n}\left(W_{p}^{r} ; L_{q}\right) \gg n^{-r /(d-1)}$, и вследствие $(2)$ $\bar{d}_{n}\left(W_{p}^{r} ; L_{q}\right) \gg n^{-r /(d-1)}$.

Установим оценки снизу в случае $1 \leqslant q<p \leqslant \infty$. Пусть задано $n \in \mathbb{N}$ и $k \in \mathbb{N}$ определено условием

$$
\operatorname{dim} \mathscr{H}\left(N_{k-1}\right) \leqslant n<\operatorname{dim} \mathscr{H}\left(N_{k}\right), \quad N_{k}=2^{k+1}-1 .
$$

Заметим, что $\operatorname{dim} \mathscr{H}(N) \asymp N^{d-1}$. Тогда, принимая во внимание теорему о проекторе для поперечника $a_{n}$ (см., например, [11]), можно записать

$$
a_{n}\left(W_{p}^{r} ; L_{q}\right) \gg a_{n}\left(W_{p}^{r} \cap \mathscr{H}\left(N_{k}\right) ; L_{q} \cap \mathscr{H}\left(N_{k}\right)\right) .
$$


Теперь аналогично случаю оценки сверху, используя теорему А, убеждаемся, что

$$
a_{n}\left(W_{p}^{r} \cap \mathscr{H}\left(N_{k}\right) ; L_{q} \cap \mathscr{H}\left(N_{k}\right)\right) \asymp N_{k}^{-r} m_{N_{k}}^{1 / p-1 / q} a_{n}\left(B_{p}^{m_{N_{k}}}\left(\alpha\left(N_{k}\right)\right) ; l_{q}^{m_{N_{k}}}\right) .
$$

Заметив, что $n \asymp m_{N_{k}} \asymp N_{k}^{d-1}$, используя результат о точных значениях поперечников $a_{k}\left(B_{p}^{m}(\alpha) ; l_{q}^{m}\right)$ при $1 \leqslant q<p \leqslant \infty[14]$ (с учетом предложения 1 ) можем записать

$$
a_{n}\left(B_{p}^{m_{N_{k}}}\left(\alpha\left(N_{k}\right)\right) ; L_{q}^{m_{N_{k}}}\right) \asymp N_{k}^{(d-1)(1 / q-1 / p)} .
$$

Объединяя соотношения (9)-(11), с учетом неравенств (2) окончательно получаем, что при $1 \leqslant q<p \leqslant \infty$ и $r>0$

$$
\bar{d}_{n}\left(W_{p}^{r} ; L_{q}\right) \gg n^{-r /(d-1)} .
$$

Теорема доказана.

\section{СПИСОК ЦИТИРОВАННОЙ ЛИТЕРАТУРЫ}

[1] И. Стейн, Г. Вейс, Введение в гармонический анализ на евклидовых пространствах, Мир, М., 1974. [2] А. Кратцер, В. Франц, Трансцендентные функции, ИЛ, М., 1963. [3] A. Bonami, J.-L. Clerk, Trans. Amer. Math. Soc., 183 (1973), 223-263. [4] Г. Ceгё, Ортогональные многочлены, Физматгиз, M., 1962. [5] R.A. DeVore, R. Howard, Ch. Micchelli, Manuscripta Math., 63:4 (1989), 469-478. [6] В. М. Тихомиров, Некоторые вопросы теории приближений, Изд-во МГУ, M., 1976. [7] Dinh Dung, Vu Quoc Thanh, Proc. Amer. Math. Soc., 124:9 (1996), 2757-2765. [8] С. М. Никольский, П. И. Лизоркин, Acta Sci. Math. (Szeged), 48:1-4 (1985), 401-416. [9] А. И. Камзолов, Матем. заметки, 32:3 (1982), 285-293. [10] Yuan Xu, Constr. Approx., 21:1 (2005), 1-28. [11] С. Н. Кудрявцев, Изв. РАН. Сер. матем., 59:4 (1995), 81-104. [12] G. Brown, D. Feng, S. Y. Sheng, J. Complexity, 18:4 (2002), 1001-1023. [13] А. И. Камзолов, УМН, 39:2 (1984), 159-160. [14] А. Б. Ходулёв, Функи. анализ и его прил., 23:2 (1989), 94-95.

B. С. Романюк

Институт математики НАН Украины

E-mail: funct@imath.kiev.ua
Поступило

02.08.2005

Исправленный вариант

11.07.2008 